\title{
Decreased expression of hyaluronan synthase 1 and 2 associates with poor prognosis in cutaneous melanoma
}

\author{
Mari Poukka', Andrey Bykachev², Hanna Siiskonen³ ${ }^{3}$ Kristiina Tyynelä-Korhonen², Päivi Auvinen², \\ Sanna Pasonen-Seppänen ${ }^{1 * \dagger}$ and Reijo Sironen ${ }^{4,5,6+}$
}

\begin{abstract}
Background: Hyaluronan is a large extracellular matrix molecule involved in several biological processes such as proliferation, migration and invasion. In many cancers, hyaluronan synthesis is altered, which implicates disease progression and metastatic potential. We have previously shown that synthesis of hyaluronan and expression of its synthases 1-2 (HAS1-2) decrease in cutaneous melanoma, compared to benign melanocytic lesions.

Methods: In the present study, we compared immunohistological staining results of HAS1 and HAS2 with clinical and histopathological parameters to investigate whether HAS1 or HAS2 has prognostic value in cutaneous melanoma. The specimens consisted of 129 tissue samples including superficial (Breslow $\leq 1 \mathrm{~mm}$ ) and deep (Breslow $>4 \mathrm{~mm}$ ) melanomas and lymph node metastases. The differences in immunostainings were analysed with non-parametric Mann-Whitney $U$ test. Associations between immunohistological staining results and clinical parameters were determined with the $x^{2}$ test. Survival between patient groups was compared by the Kaplan-Meier method using log rank test and Cox's regression model was used for multivariate analyses.
\end{abstract}

Results: The expression of HAS1 and HAS2 was decreased in deep melanomas and metastases compared to superficial melanomas. Decreased immunostaining of HAS2 in melanoma cells was significantly associated with several known unfavourable histopathologic prognostic markers like increased mitotic count, absence of tumor infiltrating lymphocytes and the nodular subtype. Furthermore, reduced HAS1 and HAS2 immunostaining in the melanoma cells was associated with increased recurrence of melanoma ( $p=0.041$ and $p=0.006$, respectively) and shortened disease- specific survival ( $p=0.013$ and $p=0.001$, respectively).

Conclusions: This study indicates that reduced expression of HAS1 and HAS2 is associated with melanoma progression and suggests that HAS1 and HAS2 have a prognostic significance in cutaneous melanoma.

Keywords: Hyaluronan, Melanoma, Hyaluronan synthases 1 and 2, Hyaluronidase 2, Prognosis, Lymph node metastasis

\section{Background}

Cutaneous melanoma is an aggressive type of skin cancer originating from pigment-producing melanocytic skin cells. The incidence of cutaneous melanoma among fair-skinned populations has risen significantly in recent decades $[1,2]$. The main risk factors for melanoma are

\footnotetext{
* Correspondence: sanna.pasonen@uef.fi

${ }^{\dagger}$ Equal contributors

${ }^{1}$ Institute of Biomedicine, University of Eastern Finland, P.O. Box 162770211

Kuopio, Finland

Full list of author information is available at the end of the article
}

ultraviolet (UV) exposure and the presence of melanocytic nevi $[3,4]$. In the early stages of the disease, cutaneous melanoma is curable with surgical excision. However, as the disease progresses, melanoma cells acquire the ability to metastasize. Cutaneous melanoma is highly metastatic, and even in the early phases of the disease there is a small subgroup of thin melanomas that develop metastases and are not able to be cured surgically. Unfortunately, there are no accurate prognostic or diagnostic biomarkers currently available to predict the progression of this disease. 
Hyaluronan is a large glycosaminoglycan residing in the extracellular matrix of most human tissues. It is expressed abundantly in normal skin, in both the epidermis and dermis. Hyaluronan is formed on the plasma membrane by three hyaluronan synthases (HAS 1-3) and during its synthesis it is discharged into the extracellular matrix. Hyaluronan, together with its primary cell surface receptor CD44, have been shown to contribute to processes necessary for cancer development such as migration, invasion and resistance to chemotherapeutic drugs [5-9]. In addition, it has been reported that hyaluronan and hyaluronan-fragments have angiogenic properties in human endothelial cells and hyaluronan contributes to wound healing and leukocyte adhesion via long hyaluronan cables [10-12].

The role of hyaluronan in melanomagenesis has remained obscure, partly due to previously published conflicting results. Some in vitro studies suggest that hyaluronan promotes melanoma cell migration and invasion $[13,14]$, while in vivo studies indicate that reduced expression of hyaluronan correlates positively with the invasiveness of cutaneous melanoma $[15,16]$. In mouse models, elevated levels of circulating hyaluronan have been shown to associate with decreased lung metastases [17]. The expression of CD44 and hyaluronan is decreased in human cutaneous melanomas and this is associated with the progression of disease and poor prognosis [15]. Our previous work showed that hyaluronan content is increased in the in situ melanomas compared to benign nevi, whereas deep melanomas (Breslow $>4 \mathrm{~mm}$ ) are almost devoid of hyaluronan [16]. Similar decreased hyaluronan content has been shown in squamous cell carcinomas (SCCs) of larynx, mouth and skin, which are tumors originating from stratified epithelia [18-20]. Decreased tumoral hyaluronan content is accompanied by an increase in the hyaluronan degrading enzyme, hyaluronidase 2 (HYAL2), and a decrease in HAS1 and HAS2 expression in invasive melanomas and lymph node metastases compared to benign nevi and in situ melanomas [16]. In contrast, hyaluronan content seems to be increased in tumors originating from simple epithelia [7]. Thus, adenocarcinomas of the breast, colorectal and ovary have abundantly hyaluronan in the tumor and stromal cells and this correlates with an unfavorable prognosis [21-23].

Our previous work showed that decreased expression of hyaluronan in the cutaneous melanoma is due to decreased expression of HAS1 and HAS2 and increased expression of HYAL2. In the present study our aim was to investigate whether HAS1-2 or HYAL2 have prognostic value for cutaneous melanoma. Here we demonstrate for the first time that decreased expression of HAS1 and HAS2 favours melanoma progression and metastasis. The immunostaining of both HAS1 and HAS2 was decreased in deeply invasive melanomas and lymph node metastases compared to superficial melanomas and this associated with several known negative prognostic factors. These tumors showed high HYAL2 immunostaining levels but interestingly, it did not affect prognosis of patients. Our work delivers new information about hyaluronan metabolism in cutaneous melanoma and identifies HAS1 and HAS2 as possible prognostic factors in this aggressive cancer.

\section{Methods}

\section{Histological samples and clinical data}

Paraffin embedded diagnostic tissue samples were taken from invasive cutaneous melanomas (thickness $<1 \mathrm{~mm}$ or $>4 \mathrm{~mm}, n=82)$ and lymph node metastases $(n=47)$ diagnosed between 1980-2010 in Kuopio University Hospital. Invasive melanomas with Breslow depths less than $1 \mathrm{~mm}$ or more than $4 \mathrm{~mm}$ were chosen to investigate the difference between the groups representing relatively different survival in general (in melanomas $<1 \mathrm{~mm}$ the 10-year survival is about $92 \%$, while in melanomas $>$ $4 \mathrm{~mm}$ it is only about $10 \%)$. The histopathological parameters were re-evaluated by an experienced histopathologist (R.S), and the clinical patient data was collected. The research has been approved by Committee on Research Ethichs of the North Savo Hospital District and The Finnish National Supervisory Authority for Welfare and Health (VALVIRA). The registry study protocol was retrospective and thus the consent of the patients for participation was not required.

\section{HAS1 and 2 and HYAL2 immunohistological stainings}

After deparaffinization, the tissue sections were cooked in $10 \mathrm{mM}$ citrate buffer ( $\mathrm{pH} \mathrm{6.0)}$ in a pressure cooker for 15 minutes and after cooling washed with $0.1 \mathrm{M}$ phosphate buffer (PB; $\mathrm{pH}$ 7.0). The endogenous peroxidase activity was blocked with $1 \% \mathrm{H}_{2} \mathrm{O}_{2}$ for 5 minutes. Thereafter the sections were washed and incubated with $1 \%$ bovine serum albumin (BSA), $0.05 \%$ Tween-20 and $0.1 \%$ Gelatin (Sigma G-2500) in PB for 30 minutes at $37{ }^{\circ} \mathrm{C}$ to block unspecific binding. After blocking, the sections were incubated with goat polyclonal antibodies for hyaluronan synthases diluted in $1 \%$ BSA (HAS1 antibody 1:100 dilution and HAS2 antibody 1:120 dilution, Santa Cruz Biotechnology, Santa Cruz, CA). HYAL2 2 was stained with rabbit polyclonal antibody, (1:100 Abcam, Cambridge, UK). In controls, the primary antibody was omitted. The specificity of the HAS and HYAL2 antibodies was tested as described in our previous work [16]. The sections were incubated at $4{ }^{\circ} \mathrm{C}$ overnight with primary antibodies. The following day, the sections were rinsed with $\mathrm{PB}$ and incubated with biotinylated secondary antibodies, anti-goat antibody (1:1000, Vector Laboratories) diluted with $1 \%$ BSA in $\mathrm{PB}$ for HASes and anti-rabbit antibody (1:200, Vector 
Laboratories) for HYAL2. The bound antibodies were visualized with avidin-biotin-peroxidase method (1:200, Vector Laboratories, Irvine, CA) using 0.05 \% 3,3-diaminobenzidine (DAB, Sigma, St.Louis, MO) as a substrate. The Mayer's hematoxylin counterstained sections were mounted in DePex (BDH Laboratory Supplies, Poole, England).

\section{Evaluation of immunohistological stainings}

The evaluation of the immunostainings was done independently by two researchers (M.P., H.S.). The immunostaining coverages and the intensities were evaluated in melanoma and stromal cells as previously described [16]. The amounts of immunopositive cells were estimated with a five-level scoring system as follows; $1=0-5 \%$, $2=6-25 \%, 3=26-50 \%, 4=51-75 \% ， 5=76-100 \%$ (Additional file 1: Figure S1). The intensities of the immunostainings were estimated with a four-level scoring system from 0 to 3 as follows; negative (0), weak (1), moderate (2) or strong (3).

\section{Statistical analyses}

Statistical analyses were performed with SPSS Statistics 21 (IBM). The differences in immunostainings between all stages (pT1, pT4 and pN1-) were analysed with nonparametric Mann-Whitney U test. Associations between immunostainings and clinical data were determined with $x^{2}$ test. For the $x^{2}$ test continuous variables were transformed into categorical variables. Univariate survival analyses of different groups were determined with Kaplan-Meier log rank test. Two Kaplan-Meier log rank test were performed to verify the accuracy of clinical data (Additional file 2: Figure S2). Multivariate analyses were performed with the Cox regression model. The multivariate analyses tests were conducted separately for two different groups because some histopathological covariates were only analysed from primary cutaneous melanoma samples (pT1 and pT4). Tests were conducted only for primary cutaneous melanomas (pT1 and pT4) without lymph node metastasis and for all stages (pT1, pT4 and pN1-). The immunostaining categories 0 $(0-5 \%)$ and 1 (6- $26 \%)$ were merged in the $x^{2}$ test, Kaplan-Meier log rank test and Cox regression model because of small group sizes. P-values less than 0.05 were considered statistically significant.

\section{Results}

Clinical information and histological samples were obtained from 129 patients; 74 males (57.4\%) and 55 (42.6\%) females (Table 1). The samples consisted of 41 superficial melanomas (Breslow $\leq 1 \mathrm{~mm}, \mathrm{pT} 1$ ), 41 deep melanomas (Breslow $>4 \mathrm{~mm}, \mathrm{pT} 4$ ) and 47 lymph node metastases of melanoma (pN1). The most common cutaneous localization of primary melanoma was the back $(26.4 \%)$. The mean age at the time of diagnosis was 59 (ranging between $5-92$ years) and the mean follow-up

Table 1 Clinical information of the patients $(n=129)$

\begin{tabular}{|c|c|c|c|c|}
\hline Variable & pT1 & pT4 & pN1- & Total \\
\hline Number of cases & $41(31.8 \%)$ & $41(31.8 \%)$ & $47(36.4 \%)$ & $129(100.0 \%)$ \\
\hline \multicolumn{5}{|l|}{ Gender } \\
\hline Male & $22(17.1 \%)$ & $24(18.6 \%)$ & $28(21.7 \%)$ & $74(57.4 \%)$ \\
\hline Female & 19 (14.7\%) & 17 (13.2\%) & 19 (14.7\%) & 55 (42.6\%) \\
\hline \multicolumn{5}{|l|}{ Age } \\
\hline under 20 & $0(0.0 \%)$ & $1(0.8 \%)$ & $1(0.8 \%)$ & $2(1.6 \%)$ \\
\hline $20-59$ & $18(14.0 \%)$ & 14 (10.9\%) & $26(20.2 \%)$ & $58(45.0 \%)$ \\
\hline 60 and over & $23(17.8 \%)$ & 26 (20.2\%) & $20(15.5 \%)$ & $69(53.5 \%)$ \\
\hline \multicolumn{5}{|l|}{ Any relapse } \\
\hline Yes & $3(2.4 \%)$ & $23(18.1 \%)$ & 39 (30.7 \%) & 65 (51.2\%) \\
\hline No & 37 (29.1\%) & $16(12.6 \%)$ & $3(2.4 \%)$ & $56(44.1 \%)$ \\
\hline Spread at diagnosis & $0(0.0 \%)$ & $1(0.8 \%)$ & $5(3.9 \%)$ & $6(4.7 \%)$ \\
\hline \multicolumn{5}{|l|}{ Alive } \\
\hline Yes & $31(24.0 \%)$ & $8(6.2 \%)$ & $9(7.0 \%)$ & $48(37.2 \%)$ \\
\hline No & $10(7.8 \%)$ & $33(25.6 \%)$ & $38(29.5 \%)$ & $81(62.8 \%)$ \\
\hline \multicolumn{5}{|l|}{ Cause of death } \\
\hline Melanoma & $1(1.2 \%)$ & $20(24.7 \%)$ & $32(39.2 \%)$ & $53(65.4 \%)$ \\
\hline Other & $5(6.2 \%)$ & $7(8.6 \%)$ & $0(0.0 \%)$ & $12(14.8 \%)$ \\
\hline Not known & 4 (4.9 \%) & 6 (7.4 \%) & 6 (7.4\%) & 16 (19.8 \%) \\
\hline
\end{tabular}


time was 8.2 years (ranging between 0.1 - 32.67 years). $71(55.0 \%)$ patients had relapse or widely metastatic disease at the time of diagnosis (Table 1). Interferon treatment, chemotherapy and radiation therapy was given to 32 (24.8\%), 36 (27.9\%) and to 41 (31.8\%) patients, respectively, with metastatic disease (data not shown).

\section{Decreased expression of HAS 1 and HAS 2 is associated with the more advanced stages of melanoma}

In superficial melanoma, melanoma cells were diffusely immunostained with both HASes (Fig. 1a, d). In melanoma cells, both the cytoplasm and plasma membrane showed immunoreactivity (up to $90 \%$ ), whereas most (up to $80 \%$ ) of the stromal cells showed no immunopositivity (Additional file 3: Figure S3). When expressed, HAS1 and HAS2 were localized in the cytoplasm and on the plasma membrane of stromal cells (Fig. 1d, insert).

A decrease in HAS1 positive melanoma cells was associated with advanced stage melanoma $(p=0.006$; Table 2 and Fig. 2). Thus, the proportion of HAS1 immunopositive melanoma cells was significantly lower in LN metastases than in superficial (pT1) melanomas ( $p=$ 0.002; Fig. 2). Similarly, the proportion of HAS2 immunopositive melanoma cells was significantly lower in deeply invasive (pT4) melanomas and LN metastases ( $\mathrm{pN} 1)(p=0.013$ and $p=0.012$, respectively) compared to superficial melanomas (Fig. 2). In addition, staining intensity of HAS1 in melanoma cells was decreased in LN metastases compared to deeply invasive melanomas $(p=0.018$, Fig. 2$)$ and HAS2 intensity in melanoma cells was decreased in deeply invasive melanomas compared to superficial ones ( $p=0.002$; Fig. 2). Decreased HAS2 intensity in melanoma cells was also associated with advanced stage ( $p=0.047$; Table 2$)$.

The overall proportion of immunopositive stromal cells was $0-5 \%$ (Additional file 3: Figure S3). Similar to melanoma cells, the strongest HAS1 and HAS2 immunostaining intensity in stromal cells was observed in superficial melanomas (Fig. 1). Decreased immunostaining intensity was observed in LN metastases compared with superficial melanomas $(\mathrm{p}=0.013$ for HAS1, $\mathrm{p}<$ 0.001 for HAS2, Additional file 3: Figure S3).

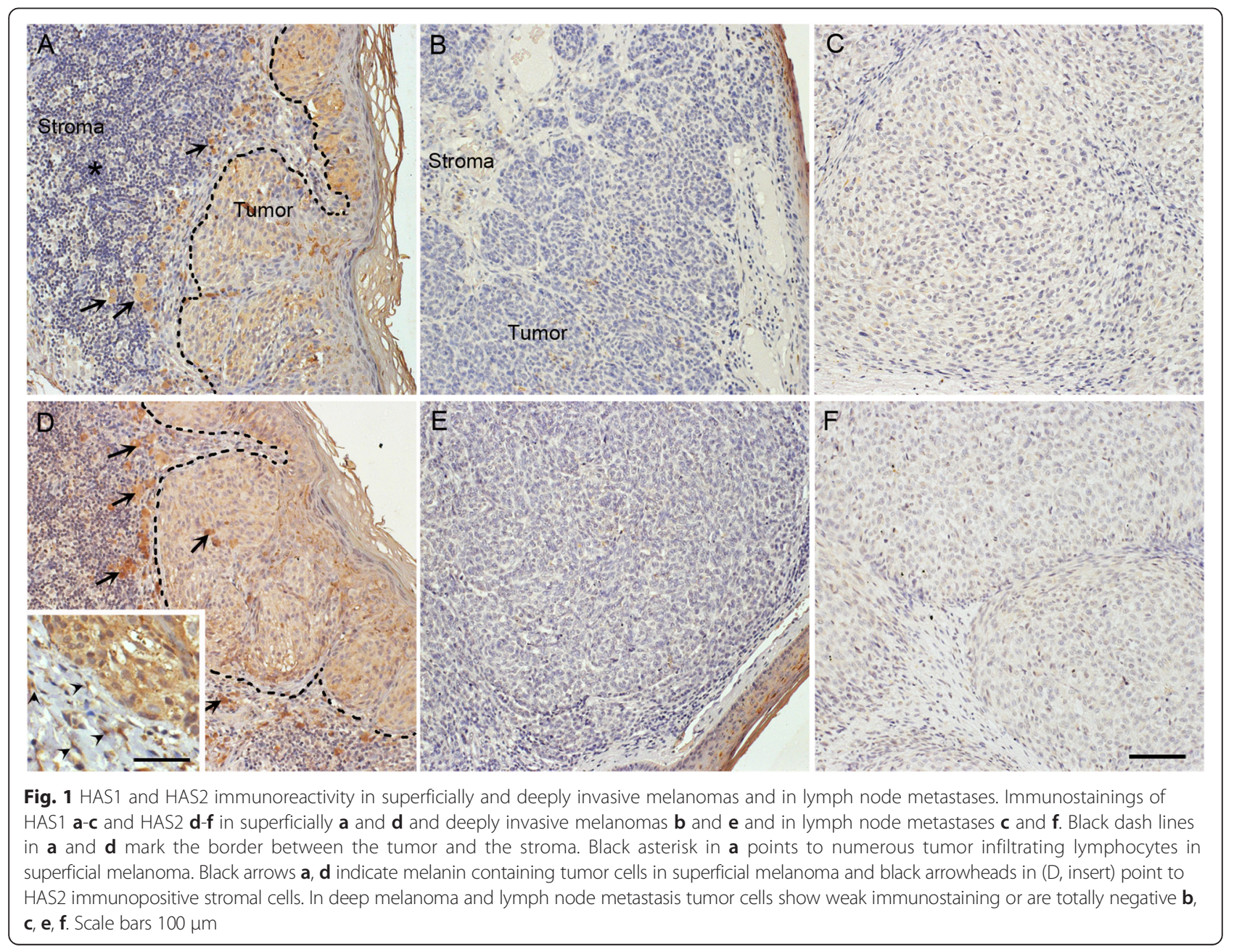


Table 2 Correlation of HAS1 and HAS2 with clinical and histopathological factors

\begin{tabular}{|c|c|c|c|c|}
\hline Variable & HAS1 Coverage & HAS1 Intensity & HAS2 Coverage & HAS2 Intensity \\
\hline Stage & $p=0.006$ & ns & ns & $p=0.047$ \\
\hline \multicolumn{5}{|l|}{ pT1, pT4 or pN1- } \\
\hline Melanoma related death & $p=0.007$ & ns & $p=0.001$ & $p=0.016$ \\
\hline Recurrence & $p=0.021$ & ns & $p=0.007$ & ns \\
\hline Regional recurrence & $p=0.006$ & $p=0.023$ & $p=0.007$ & ns \\
\hline Distant recurrence & $p=0.012$ & ns & $p=0.001$ & $p=0.004$ \\
\hline Genre & ns & ns & ns & ns \\
\hline Age & ns & ns & ns & ns \\
\hline Ulceration & ns & ns & ns & ns \\
\hline TIL (low / moderate / high) & ns & ns & $p=0.036$ & $p=0.040$ \\
\hline Mitotic count (mitosis/mm²) & ns & ns & ns & $p=0.018$ \\
\hline Horizontal diameter of melanoma (mm) & ns & ns & $p=0.002$ & $p=0.042$ \\
\hline \multicolumn{5}{|l|}{ Growth type } \\
\hline Nodular & ns & ns & ns & $p=0.001$ \\
\hline
\end{tabular}

Stage $=\mathrm{pT1}$, pT4 or pN1.- TL = tumor-infiltrating lymphocytes (evaluated either low, moderate or high amount). Mitosis = mitosis/mm2, horizontal tumor diameter $(\mathrm{mm})$

\section{Low HAS1 and HAS 2 expression is associated with melanoma related death}

Decreased coverage of HAS2 immunostaining in melanoma cells was associated with several histopathological factors, including reduced number of tumor infiltrating lymphocytes (TILs) $(p=0.036)$ and increased horizontal tumor diameter ( $p=0.002$; Table 2). Results were similar for the intensity of HAS2 immunostaining; lower HAS2 intensity was associated with a reduced number of TILs $(p=0.040)$, a larger horizontal diameter $(p=0.042)$, nodular subtype $(p=0.001)$ and an increased mitotic activity ( $p=0.018$; Table 2 ). On the other hand, increased intensity of HAS2 in melanoma cells was associated with superficial type $(p=0.047$; data not shown). Neither coverage nor intensity of HAS1 staining associated with any histopathological factors (Table 2). Reduced HAS2 immunostaining (coverage and intensity) was associated with melanoma-related death $(p=0.001$ and $p=0.016$, respectively; Table 2). Furthermore, decreased HAS1 coverage in melanoma cells ( $p=0.007$; Table 2$)$, and decreased intensity of HAS2 in the stromal cells, was positively associated with melanoma-related death $(p=0.038$; data not shown). Reduced coverage of HAS1 and HAS2 in melanoma cells was associated with recurrence of the disease, both regional and distant $(p=0.021$ and $p=$ 0.007 , respectively; Table 2 recurrence). Increased regional recurrence was related to reduced number of HAS1 and HAS2 -positive melanoma cells $(p=0.006$ and $p=0.007$, respectively; Table 2 regional and distant recurrence). Similarly, increased distant recurrence was related to reduced HAS1 and HAS2 positive melanoma cells $(p=0.012$ and $p=0.001$, respectively; Table 2$)$. Decreased intensity of HAS1 in melanoma cells was related to increased regional metastasis ( $p=0.023$; Table 2), while decreased intensity of HAS2 was associated with distant metastasis $(p=0.004$; Table 2).

\section{Reduced expression of HAS 1 and HAS 2 is associated with decreased disease-specific survival}

At the end of the follow-up time, 48 patients were alive and 81 had deceased. In melanoma cells, a reduced amount of HAS1 positivity was associated with decreased disease-specific survival (DSS) ( $p=0.013$; Fig. 3) and recurrence-free survival (RFS) ( $p=0.041$, data not shown). Similarly, decreased HAS2 coverage in melanoma cells was associated with poorer DSS ( $p=0.001$; Fig. 3$)$ and RFS ( $p=0.006$; Fig. 3$)$, and decreased intensity of HAS2 staining was related to shortened DSS ( $p=0.014$; Fig. 3$)$. In contrast, HAS1 intensity in melanoma cells was not associated with DSS. In stromal cells, HAS1 staining was not associated with either DSS or RFS, while decreased intensity of HAS2 was associated with poorer DSS $(p=0.049$, data not shown) and RFS ( $p=0.008$, data not shown).

Multivariate analyses were done in two different ways; for the primary cutaneous melanomas only (pT1 and pT4) and for all stages (pT1, pT4 and pN1). Covariates used in cutaneous melanomas (pT1 and pT4) were: Breslow's classification, ulceration, mitotic rate, patients age and immunostaining results of HAS1 and HAS2. Significant adverse prognostic factors for decreased DSS were increased Breslow's depth $(p=0.001)$ and decreased HAS1 and HAS2 staining intensity in melanoma cells $(p=0.019$ and $p=0.011$, respectively). For RFS, significant adverse prognostic factors were deep invasion $(p<0.001)$ and decreased HAS2 staining intensity of melanoma cells $(p=0.014)$. 


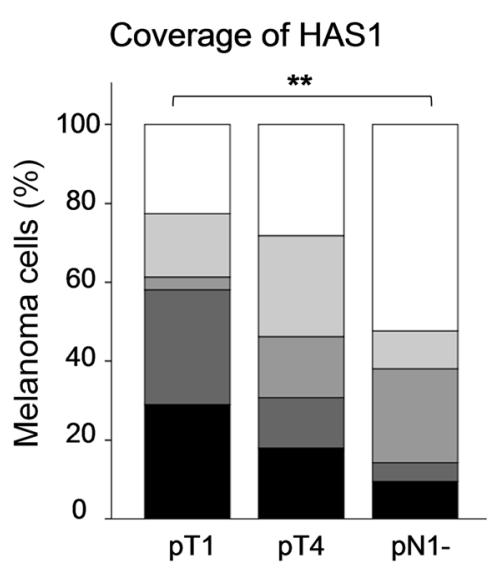

\section{Intensity of HAS1}

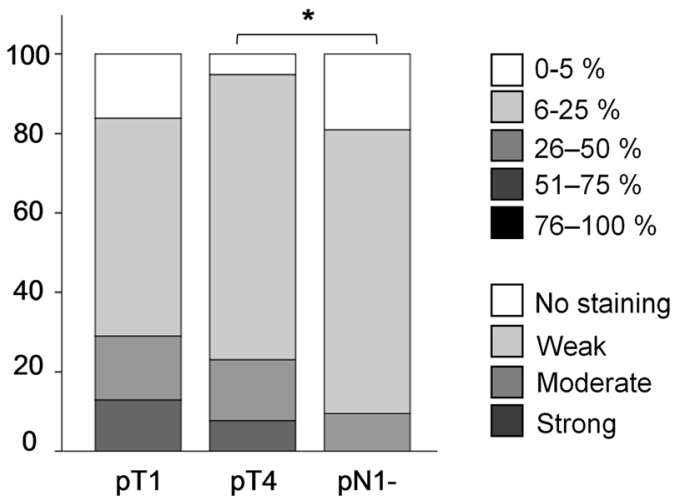

Intensity of HAS2
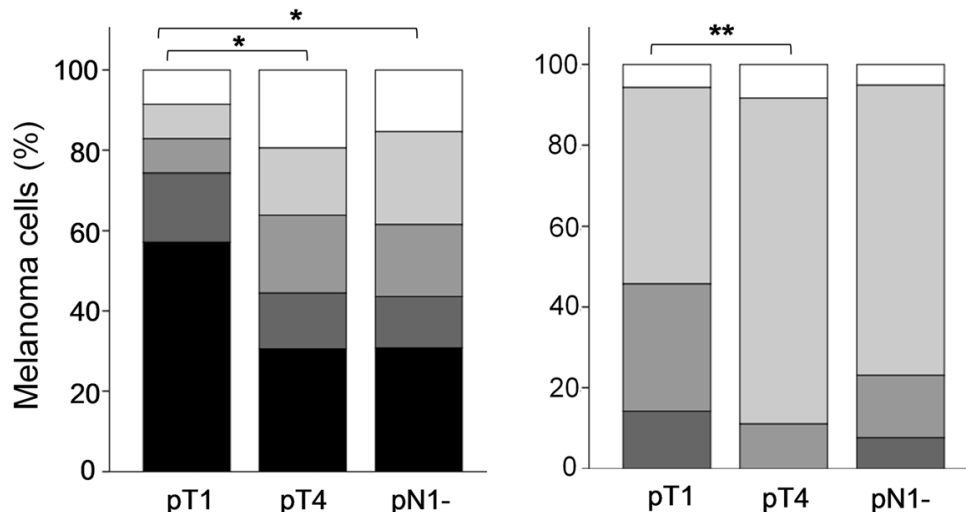

Fig. 2 Coverage and intensity of HAS1 and HAS2 immunostainings in melanoma cells of superficial melanoma (pT1), deep melanoma (pT4) and lymph node metastasis (pN1-). Coverage and intensity of HAS1 immunostainings were successfully recorded from 112 samples. Coverage and intensity of HAS2 immunostainings were recorded from 110 samples. Statistically significant differences between the stages are indicated with brackets (Mann-Whitney $U$ test). ${ }^{*} p$-value $<0.05$, ${ }^{* *} p$-value $<0.01$, ${ }^{* * *} p$-value $<0.001$

Covariates used in multivariate analyses for all stages (pT1, pT4 and pN1) were: patients' age, stage (pT1, pT4 and pN1) and immunostaining results of HAS1 and HAS2. Decreased coverage of HAS2 positive melanoma cells was a significant negative prognostic factor $(p=$ $0.039)$ for DSS, similar to increased stage $(p=0.001)$ and age $(p=0.035)$. HAS1 immunostaining did not have prognostic value for DSS.

\section{Expression of Hyaluronidase 2 in melanoma}

HYAL2 immunostaining localized mostly on the cytoplasm of the melanoma cells (Fig. 4). The proportion of HYAL2 positive melanoma cells was mostly high (76-100 \%, data not shown) for all stages. Immunostaining intensities of HYAL2 were statistically uniform in all stages (Fig. 4). Between 50-60\% of samples had weak intensity in all stages (data not shown).

Coverage of stromal immunostaining was between 0-50\% with no statistical differences between stages, and there were no differences in the intensities of stromal staining. In all stages, the staining intensities of stromal cells were either weak or there were no staining.

\section{Discussion}

The present work demonstrates that reduced expression of HAS1 and HAS2 is associated with an unfavorable prognosis in cutaneous melanoma. Reduced expression of HAS1 and HAS2 is significantly associated with reduced DSS and RFS. In addition, weak immunostaining of HAS2 in melanoma cells is associated with unfavorable histopathologic prognostic markers such as increased mitotic count, absence of tumor infiltrating lymphocytes and nodular subtype. Furthermore, multivariate analysis indicates that decreased expression of HAS1 and HAS2 in melanoma cells are independent prognostic factors.

Decreased tumoral hyaluronan content has been shown to be an adverse prognostic factor in cutaneous melanoma [15]. We have previously demonstrated that decreased expression of HAS1 and HAS2 and increased 

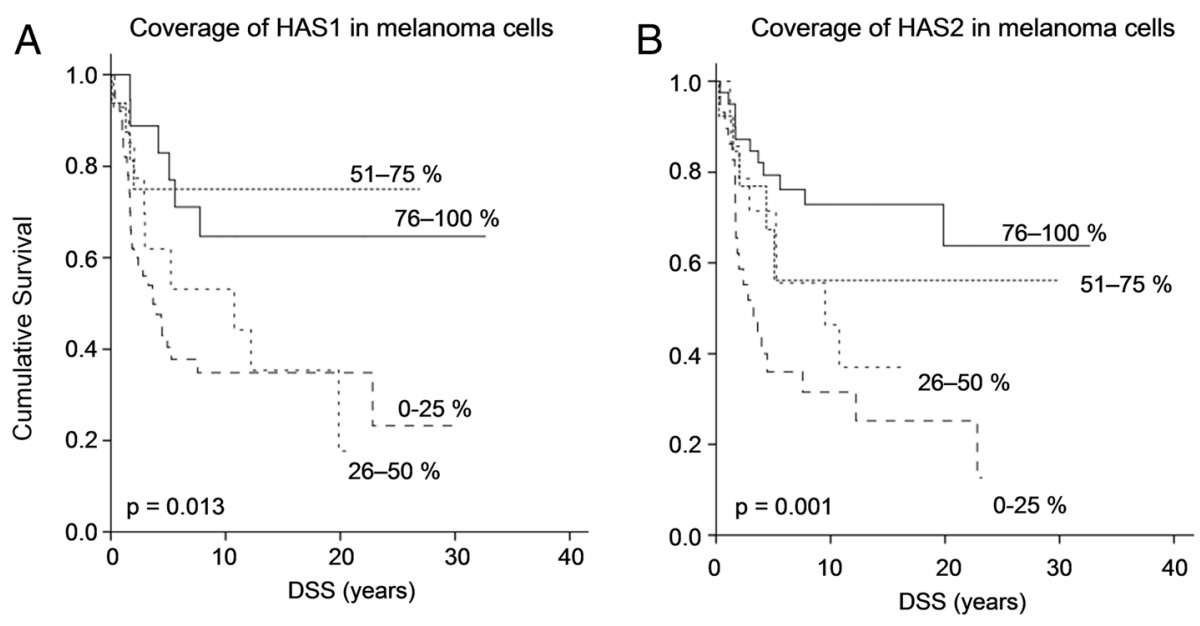

C Intensity of HAS2 in melanoma cells
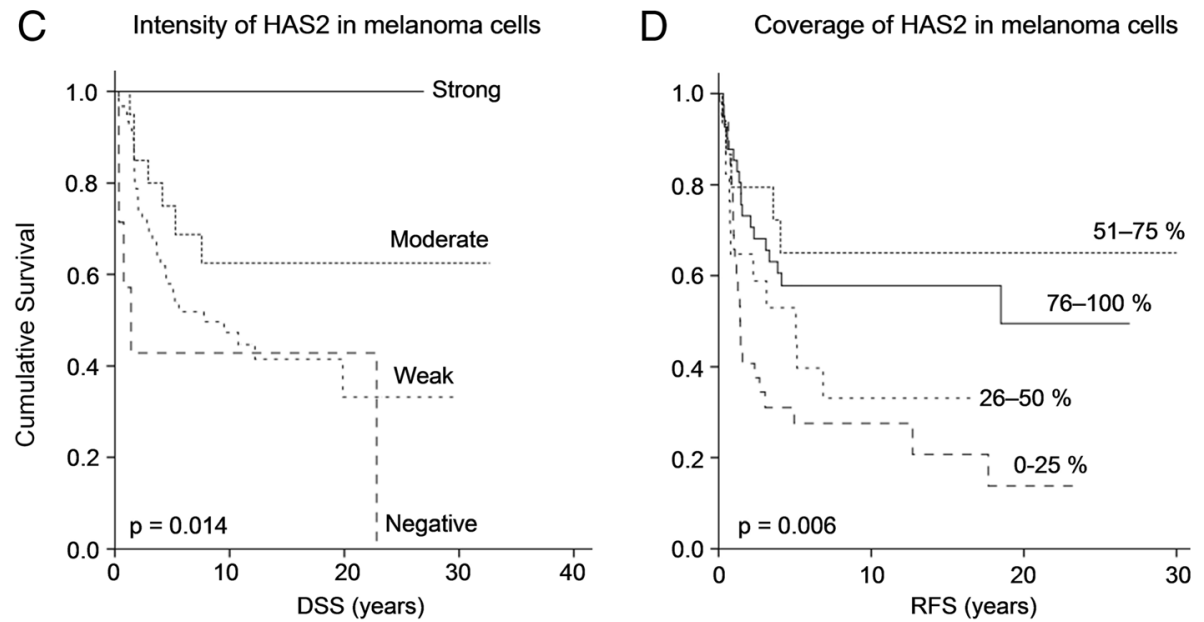

Fig. 3 Kaplan-Meier survival curves according to HAS1 and HAS2 expression. Kaplan-Meier log rank test indicating association of decreased HAS1 $\mathbf{a}$ and HAS2 $\mathbf{b}$ coverage and decreased intensity of HAS2 c in melanoma cells with declined disease-specific survival. Kaplan-Meier log rank test indicating association of decreased HAS2 coverage with declined recurrence-free survival $\mathbf{d}$. DSS = disease-specific survival, RFS = recurrence-free survival

expression of hyaluronan degrading enzyme HYAL2 correlates with decreased tumoral hyaluronan content in the invasive melanomas [16]. In the present work, we showed that decreased expression of HAS1 and HAS2 are adverse prognostic factors, while the expression of
HYAL2 does not affect the prognosis. Previously we showed that HYAL2 expression is elevated in dysplastic nevi and the expression is also elevated in locally invasive and metastatic melanomas [16]. Whereas the expression of HAS1 and HAS2 correlated with the content of
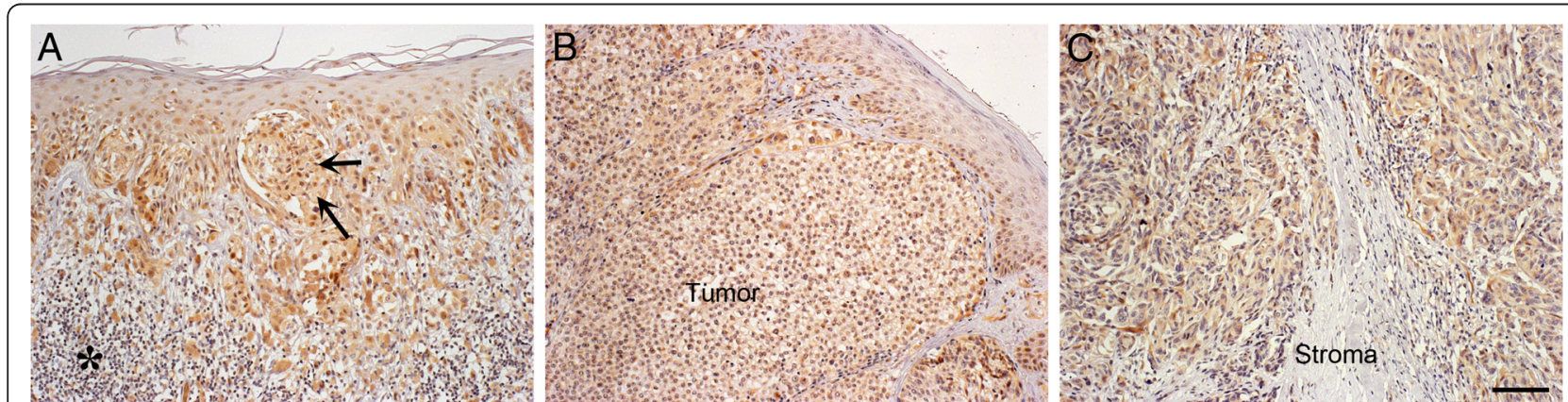

Fig. 4 HYAL2 immunostaining in superficially $\mathbf{a}$ and deeply invasive melanomas $\mathbf{b}$ and in lymph node metastases $\mathbf{c}$. Black asterisk in a points to numerous tumor infiltrating lymphocytes and black arrows immunopositive tumor cells in superficial melanoma. Scale bar $100 \mu \mathrm{m}$ 
hyaluronan in tumor tissue, and their expressions are not altered until the invasive phase of the disease, at which time hyaluronan content decreased [16]. This suggests that HASes are responsible for intratumoral hyaluronan concentration and they may have an adverse impact on tumor progression by modulating hyaluronan content in the tumor tissue.

Several melanoma cell lines synthesize substantial amounts of hyaluronan in vitro [24, 25]. Furthermore, melanoma cell-derived factors are able to induce hyaluronan synthesis in cutaneous fibroblasts via upregulation of HAS2 [26]. These findings suggest that in melanoma hyaluronan is produced by both melanoma and stromal cells, but most likely the majority of intratumor hyaluronan originates from melanoma cells. Our previous work included in situ melanomas, which expressed excessively hyaluronan [16]. Since in situ melanomas localize in epidermis without any proper stromal component, it is possible that the most of hyaluronan in these tumors originates from melanoma cells and also from epidermal keratinocytes, which are known to express all HASes [27]. In addition to hyaluronan, several other extracellular matrix molecules are also shown to be involved in melanomagenesis like versican and fibronectin [28-30]. Silencing of versican increases cell proliferation and migration, whereas silencing of fibronectin increases drug sensitivity of melanoma cells $[28,30]$.

Hyaluronan metabolism in cutaneous melanoma seems to differ from the main adenocarcinomas, such as the breast carcinoma. Increased stromal hyaluronan content has been associated with poor survival and tumor differentiation in various human adenocarcinomas, whereas reduced levels of hyaluronan are associated with worsened survival in melanoma and squamous cell carcinomas (SCC) of the larynx, mouth and skin [15, 18-20]. Normal skin, both epidermis and dermis, contains extensive amounts of hyaluronan. Interestingly, the hyaluronan concentration is further increased in the in situ phase of melanoma [16]. This increase in the early phase lesions has also been observed in cutaneous, laryngeal and oral SCCs $[16,18,19,31]$. The results suggest that loss of hyaluronan is associated with the acquisition of a motile, invasive tumor cell phenotype. Increased content of hyaluronan may reflect an attempt to maintain hyaluronan synthesis at levels that are normal for the respective tissues. Physiologically, hyaluronan acts as a protective barrier against harmful substances, microbes and UV radiation. Rauhala showed that UVB exposure in keratinocytes causes increased hyaluronan synthesis via up-regulation of HAS13 , which may have a protective effect on cells by increasing viability and decreasing the secretion of inflammatory mediators [27, 32]. Decrease of hyaluronan content in invasive melanoma and SCCs may increase invasiveness of the tumor cells, which is in agreement with recent findings where the accumulation of high molecular mass hyaluronan exerted anticancer like effects in naked mole rats [33]. This phenomenon was related to the exceptionally large molecular size of hyaluronan in these animals. Indeed, activation of hyaluronan degrading enzyme, HYAL2, led to a reduction in the high molecular mass hyaluronan, which resulted in tumor promotion in this model [33]. Moreover, our unpublished in vitro observations support the idea that hyaluronan overexpression tends to restrict melanoma cell growth, and melanoma cell lines (MV3 and C8161) overexpressing HAS3 show reduced cell motility and proliferation [25].

Knowledge of the prognostic significance of hyaluronan synthases in malignant tumors is currently relatively limited. In contrast to melanoma, increased HAS1-3 immunoreactivity is associated with poor survival in breast cancer [34]. In particular, HAS2 has been shown to suppress tissue metalloproteinase inhibitor 1 which increases the invasiveness of breast cancer cells [35]. Furthermore, increased transcription levels of HAS1 and HYAL1 are associated with metastasizing urothelial bladder carcinoma [36]. The prognostic value of a reduced HAS1-2 expression likely comes from decreased synthesis of hyaluronan. However, the finding that HAS1 and 2 are independent prognostic factors in melanoma raises the possibility that these enzymes by themselves affect tumor progression. For example, our unpublished in vitro observations indicate that cell adhesion is reduced in melanoma cells overexpressing HAS3 and this ability are not reversed with eradication of hyaluronan [25]. These results suggest hyaluronan synthesizing enzymes may independently affect cell function in the absence of any direct effects on hyaluronan synthesis.

Our results demonstrate that the proportion of HAS1 and HAS2 and hyaluronan positive melanoma cells is significantly decreased in lymph node metastases, compared with superficially invasive melanoma. These results indicate that decreased expression of HAS1 and HAS2, and thus reduced tumoral hyaluronan content, is a favorable feature for metastatic melanoma cells. Similarly, ovarian carcinoma cells synthesizing low amounts of hyaluronan were most adherent to the intra-abdominal peritoneal surfaces, suggesting that a large pericellular hyaluronan coat acts as a barrier for adhesion and inhibits peritoneal dissemination [37]. In addition to HAS1 and HAS2 expression, the observed HYAL2 expression may contribute to melanoma progression. The presence of hyaluronidase and hyaluronan fragments produced by hyaluronidases has been shown to mediate tumor progression by stimulating angiogenesis and tumor invasion [38, 39]. HYAL2 degrades hyaluronan to oligosaccharides, which may induce cleavage of the main cell surface hyaluronan receptor, CD44, resulting in increased motility and 
invasion [40]. HYAL2 has also been shown to directly cleave $\mathrm{CD} 44$, which may disturb the hyaluronan-CD44 interaction and release locally growing melanoma cells enabling the cells to spread [41]. In addition to CD44 shedding, the expression of certain CD44 variants has been shown to induce disease dissemination [42, 43]. Indeed, the expression of receptor variant CD44v6 associates strongly with brain metastases [42].

Our results indicate that reduced immunopositivity of HAS2 is associated with several known unfavorable histopathologic prognostic markers like a reduced number of tumor infiltrating lymphocytes (TIL), an increased melanoma horizontal diameter, an increased mitotic activity and the nodular subtype. The proinflammatory effect of hyaluronan has been previously comprehensively demonstrated $[10,44,45]$. The presence of hyaluronan depositions, and the formation of hyaluronan cables, recruits leukocytes to the site of inflammation and leukocytes binding to these cables occur mainly via CD44 [46, 47]. Interaction of hyaluronan-CD44 is important in numerous inflammatory diseases, such as allergic dermatitis and inflammatory liver disease [48-50]. In melanoma, hyaluronan may increase leukocyte infiltration, and therefore, the loss of hyaluronan could contribute to a reduction in TILs, thereby worsening the prognosis $[51,52]$. This is an interesting finding since the majority of new therapies in metastatic melanoma operate through activation of immune responses [53].

\section{Conclusions}

Caught in its early stages, melanoma can be cured by surgery. However, despite a recent surge in the development of new targeted therapies, metastatic melanoma remains a major challenge to treat. Our novel data provides novel information about hyaluronan metabolism in cutaneous melanoma and points towards a significant role for HAS1 and HAS2 in melanoma dissemination. Our results about correlation between decreased immunostaining of HAS1 and HAS2 and decreased survival of patients support the previous works and bring us new information about histopathological changes that happen during melanoma progression. However, whether decreased expression of HAS1 and HAS2 is the cause or a secondary consequence of cutaneous melanoma is a question that awaits further investigation.

\section{Additional files}

Additional file 1: Figure S1. Evaluation of immunopositivity using fivelevel scoring system. $\mathbf{a}$ and $\mathbf{b}$ representing $0-5 \%$ of melanoma cells stained positively, c representing 6-25\% of melanoma cells stained positively, $\mathbf{d}$ representing 26-50\% of melanoma cells stained positively, e representing 51-75\% melanoma cells stained positively and $\mathbf{f}$ representing $76-100 \%$ of melanoma cells stained positively. Scale bar $100 \mu \mathrm{m}$. (TIF 19623 kb)
Additional file 2: Figure S2. Clinical data's accuracy was verified with two Kaplan-Meier log rank tests. Kaplan-Meier log rank test according stage (pT1, pT4 and pN1-) a. Patients with pT1 melanoma had better prognosis than patients with PT4 melanoma or lymph node metastasis $(p<0.001)$. Kaplan-Meier log rank test according tumor-infiltrating lymphocytes -status $\mathbf{b}$. Tumor infiltrating lymphocytes status of pT1 and pT4 melanomas were analyzed and higher amounts of tumorinfiltrating lymphocytes associated with better prognosis $(p=0.006)$. DSS = disease-specific survival. (TIF $536 \mathrm{~kb}$ )

Additional file 3: Figure S3. Immunostaining results of HAS1 and HAS2 in stromal cells. Coverage and intensity of HAS1 and HAS2 immunostainings in the stroma of superficial melanoma (pT1), deep melanoma (pT4) and lymph node metastasis (pN1-). Coverage and intensity of HAS1 immunostainings were recorded from 95 and 96 samples, respectively. Coverage and intensity of HAS2 immunostainings were recorded from 90 samples. Statistically significant differences between the stages are indicated with brackets (Mann-Whitney U test). ${ }^{*} p$-value $<0.05$, ${ }^{* *} p$-value $<0.01$, *** $p$-value $<0.001$. (TIF $1091 \mathrm{~kb}$ )

\section{Abbreviations}

HA: Hyaluronan; HAS: Hyaluronan synthase; HYAL: Hyaluronidase; DSS: Disease specific survival; RFS: Recurrence free survival; LN: Lymph node; PB: Phosphate buffer; BSA: Bovine serum albumin.

\section{Competing interests}

The authors declare that they have no competing interests.

\section{Authors' contribution}

MP analyzed histopathological specimens, participated on the collection of the clinical data, performed the statistical analyses and drafted the manuscript. $A B$ participated on the collection of the clinical data and commented on the manuscript. HS analyzed histopathological specimens and commented on the manuscript. KTK organized clinical data collection and commented on the manuscript. PA advised and helped performing the statistical analyses and commented on the manuscript. SPS designed this study and the immunohistological stainings, coordinated the study and helped to draft the manuscript. RS designed this study, analyzed histopathological specimens and helped to draft the manuscript. All authors read and approved the final manuscript.

\section{Acknowledgements}

The authors greatly acknowledge Mrs. Eija Rahunen and Mr. Kari Kotikumpu for excellent technical assistance. Financial support for this work was provided by The Academy of Finland, the Special Government Funding of Kuopio University Hospital, The Spearhead Funds of the University of Eastern Finland/Cancer Center of Eastern Finland and the Finnish Medical Society Duodecim.

\section{Author details}

${ }^{1}$ Institute of Biomedicine, University of Eastern Finland, P.O. Box 162770211 Kuopio, Finland. ${ }^{2}$ Cancer Center, Kuopio University Hospital, Kuopio, Finland. ${ }^{3}$ Department of Dermatology, University of Eastern Finland and Kuopio University Hospital, Kuopio, Finland. ${ }^{4}$ Institute of Clinical Medicine/Clinical Pathology, University of Eastern Finland, Kuopio, Finland. ${ }^{5}$ Department of Clinical Pathology, Kuopio University Hospital, Kuopio, Finland. ${ }^{6}$ Cancer Center of Eastern Finland, Kuopio, Finland.

Received: 8 July 2015 Accepted: 8 May 2016

Published online: 16 May 2016

\section{References}

1. Geller AC, Clapp RW, Sober AJ, Gonsalves L, Mueller L, Christiansen CL, Shaikh W, Miller DR. Melanoma epidemic: an analysis of six decades of data from the Connecticut Tumor Registry. J Clin Oncol. 2013;31(33):4172-8.

2. Jemal A, Saraiya M, Patel P, Cherala SS, Barnholtz-Sloan J, Kim J, Wiggins CL, Wingo PA. Recent trends in cutaneous melanoma incidence and death rates in the United States, 1992-2006. J Am Acad Dermatol. 2011;65(5 Suppl 1):S17-25.e1-3. 
3. Lin WM, Luo S, Muzikansky A, Lobo AZ, Tanabe KK, Sober AJ, Cosimi AB, Tsao H, Duncan LM. Outcome of patients with de novo versus nevus-associated melanoma. J Am Acad Dermatol. 2015;72(1):54-8.

4. Moan J, Grigalavicius M, Baturaite Z, Dahlback A, Juzeniene A. The relationship between UV exposure and incidence of skin cancer. Photodermatol Photoimmunol Photomed. 2015;31(1):26-35.

5. Kim HR, Wheeler MA, Wilson CM, lida J, Eng D, Simpson MA, McCarthy JB, Bullard KM. Hyaluronan facilitates invasion of colon carcinoma cells in vitro via interaction with CD44. Cancer Res. 2004;64(13):4569-76.

6. Misra S, Ghatak S, Zoltan-Jones A, Toole BP. Regulation of multidrug resistance in cancer cells by hyaluronan. J Biol Chem. 2003;278(28):25285-8.

7. Sironen RK, Tammi M, Tammi R, Auvinen PK, Anttila M, Kosma VM. Hyaluronan in human malignancies. Exp Cell Res. 2011;317(4):383-91.

8. Tammi RH, Kultti A, Kosma VM, Pirinen R, Auvinen P, Tammi MI. Hyaluronan in human tumors: pathobiological and prognostic messages from cellassociated and stromal hyaluronan. Semin Cancer Biol. 2008;18(4):288-95.

9. Wang SJ, Bourguignon LY. Hyaluronan and the interaction between CD44 and epidermal growth factor receptor in oncogenic signaling and chemotherapy resistance in head and neck cancer. Arch Otolaryngol Head Neck Surg. 2006;132(7):771-8.

10. de la Motte CA, Hascall VC, Drazba J, Bandyopadhyay SK, Strong SA Mononuclear leukocytes bind to specific hyaluronan structures on colon mucosal smooth muscle cells treated with polyinosinic acid:polycytidylic acid: inter-alpha-trypsin inhibitor is crucial to structure and function. Am J Pathol. 2003;163(1):121-33.

11. Lokeshwar VB, Selzer MG. Differences in hyaluronic acid-mediated functions and signaling in arterial, microvessel, and vein-derived human endothelial cells. J Biol Chem. 2000;275(36):27641-9.

12. Tammi R, Pasonen-Seppanen S, Kolehmainen E, Tammi M. Hyaluronan synthase induction and hyaluronan accumulation in mouse epidermis following skin injury. J Invest Dermatol. 2005;124(5):898-905.

13. Edward M, Quinn JA, Pasonen-Seppanen SM, McCann BA, Tammi RH. 4-Methylumbelliferone inhibits tumour cell growth and the activation of stromal hyaluronan synthesis by melanoma cell-derived factors. $\mathrm{Br} J$ Dermatol. 2010;162(6):1224-32.

14. Ichikawa T, Itano N, Sawai T, Kimata K, Koganehira Y, Saida T, Taniguchi S. Increased synthesis of hyaluronate enhances motility of human melanoma cells. J Invest Dermatol. 1999;113(6):935-9.

15. Karjalainen JM, Tammi RH, Tammi MI, Eskelinen MJ, Agren UM, Parkkinen JJ, Alhava EM, Kosma VM. Reduced level of CD44 and hyaluronan associated with unfavorable prognosis in clinical stage I cutaneous melanoma. Am J Pathol. 2000;157(3):957-65.

16. Siiskonen H, Poukka M, Tyynela-Korhonen K, Sironen R, Pasonen-Seppanen S. Inverse expression of hyaluronidase 2 and hyaluronan synthases $1-3$ is associated with reduced hyaluronan content in malignant cutaneous melanoma. BMC Cancer. 2013;13:181-2407-13-181.

17. Hirose Y, Saijou E, Sugano Y, Takeshita F, Nishimura S, Nonaka H, Chen YR, Sekine K, Kido T, Nakamura T, Kato S, Kanke T, Nakamura K, Nagai R, Ochiya T, Miyajima A. Inhibition of Stabilin-2 elevates circulating hyaluronic acid levels and prevents tumor metastasis. Proc Natl Acad Sci U S A. 2012; 109(11):4263-8.

18. Hirvikoski P, Tammi R, Kumpulainen E, Virtaniemi J, Parkkinen JJ, Tammi M, Johansson R, Agren U, Karhunen J, Kosma VM. Irregular expression of hyaluronan and its CD44 receptor is associated with metastatic phenotype in laryngeal squamous cell carcinoma. Virchows Arch. 1999:434(1):37-44.

19. Karvinen S, Kosma VM, Tammi MI, Tammi R. Hyaluronan, CD44 and versican in epidermal keratinocyte tumours. Br J Dermatol. 2003;148(1):86-94.

20. Kosunen A, Ropponen K, Kellokoski J, Pukkila M, Virtaniemi J, Valtonen H, et al. Reduced expression of hyaluronan is a strong indicator of poor survival in oral squamous cell carcinoma. Oral Oncol. 2004;40(3):257-63.

21. Anttila MA, Tammi RH, Tammi MI, Syrjanen KI, Saarikoski SV, Kosma VM. High levels of stromal hyaluronan predict poor disease outcome in epithelial ovarian cancer. Cancer Res. 2000;60(1):150-5.

22. Auvinen P, Tammi R, Parkkinen J, Tammi M, Agren U, Johansson R, Hirvikoski P, Eskelinen M, Kosma VM. Hyaluronan in peritumoral stroma and malignant cells associates with breast cancer spreading and predicts survival. Am J Pathol. 2000;156(2):529-36.

23. Ropponen K, Tammi M, Parkkinen J, Eskelinen M, Tammi R, Lipponen P, Agren U, Alhava E, Kosma VM. Tumor cell-associated hyaluronan as an unfavorable prognostic factor in colorectal cancer. Cancer Res. 1998; 58(2):342-7.
24. Kultti A, Pasonen-Seppanen S, Jauhiainen M, Rilla KJ, Karna R, Pyoria E, Tammi RH, Tammi Ml. 4-Methylumbelliferone inhibits hyaluronan synthesis by depletion of cellular UDP-glucuronic acid and downregulation of hyaluronan synthase 2 and 3. Exp Cell Res. 2009:315(11):1914-23.

25. Takabe P, Bart G, Ropponen A, Rilla K, Tammi M, Tammi R, PasonenSeppanen S. Hyaluronan synthase 3 (HAS3) overexpression downregulates MV3 melanoma cell proliferation, migration and adhesion. Exp Cell Res. 2015;337(1):1-15.

26. Pasonen-Seppanen S, Takabe P, Edward M, Rauhala L, Rilla K, Tammi M, Tammi R. Melanoma cell-derived factors stimulate hyaluronan synthesis in dermal fibroblasts by upregulating HAS2 through PDGFR-PI3K-AKT and p38 signaling. Histochem Cell Biol. 2012;138(6):895-911.

27. Rauhala L, Hamalainen L, Salonen P, Bart G, Tammi M, Pasonen-Seppanen S, et al. Low dose ultraviolet $B$ irradiation increases hyaluronan synthesis in epidermal keratinocytes via sequential induction of hyaluronan synthases Has1-3 mediated by p38 and Ca2+/calmodulin-dependent protein kinase II (CaMKII) signaling. J Biol Chem. 2013;288(25):17999-8012.

28. Hernandez D, Miquel-Serra L, Docampo MJ, Marco-Ramell A, Bassols A. Role of versican V0/N1 and CD44 in the regulation of human melanoma cell behavior. Int J Mol Med. 2011;27(2):269-75.

29. Bu P, Yang P. MicroRNA-203 inhibits malignant melanoma cell migration by targeting versican. Exp Ther Med. 2014;8(1):309-15.

30. Afasizheva A, Devine A, Tillman H, Fung KL, Vieira WD, Blehm BH, et al. Mitogen-activated protein kinase signaling causes malignant melanoma cells to differentially alter extracellular matrix biosynthesis to promote cell survival. BMC Cancer. 2016;16(1):186-016-2211-7.

31. Wang C, Tammi M, Guo H, Tammi R. Hyaluronan distribution in the normal epithelium of esophagus, stomach, and colon and their cancers. Am J Pathol. 1996;148(6):1861-9.

32. Hasova M, Crhak T, Safrankova B, Dvorakova J, Muthny T, Velebny V, Kubala L. Hyaluronan minimizes effects of UV irradiation on human keratinocytes. Arch Dermatol Res. 2011;303(4):277-84.

33. Tian X, Azpurua J, Hine C, Vaidya A, Myakishev-Rempel M, Ablaeva J, et al. High-molecular-mass hyaluronan mediates the cancer resistance of the naked mole rat. Nature. 2013;499(7458):346-9.

34. Auvinen $P$, Rilla $K$, Tumelius $R$, Tammi M, Sironen $R$, Soini $Y$, et al. Hyaluronan synthases (HAS1-3) in stromal and malignant cells correlate with breast cancer grade and predict patient survival. Breast Cancer Res Treat. 2014;143(2):277-86.

35. Bernert B, Porsch H, Heldin P. Hyaluronan synthase 2 (HAS2) promotes breast cancer cell invasion by suppression of tissue metalloproteinase inhibitor 1 (TIMP-1). J Biol Chem. 2011;286(49):42349-59.

36. Kramer MW, Escudero DO, Lokeshwar SD, Golshani R, Ekwenna OO, Acosta $\mathrm{K}$, et al. Association of hyaluronic acid family members (HAS1, HAS2, and HYAL-1) with bladder cancer diagnosis and prognosis. Cancer. 2011;117(6): 1197-209.

37. Tamada Y, Takeuchi H, Suzuki N, Aoki D, Irimura T. Cell surface expression of hyaluronan on human ovarian cancer cells inversely correlates with their adhesion to peritoneal mesothelial cells. Tumour Biol. 2012;33(4):1215-22.

38. Fieber C, Baumann P, Vallon R, Termeer C, Simon JC, Hofmann M, et al. Hyaluronan-oligosaccharide-induced transcription of metalloproteases. J Cell Sci. 2004;117(Pt 2):359-67.

39. Rodgers LS, Lalani S, Hardy KM, Xiang X, Broka D, Antin PB, Camenisch TD. Depolymerized hyaluronan induces vascular endothelial growth factor, a negative regulator of developmental epithelial-to-mesenchymal transformation. Circ Res. 2006;99(6):583-9.

40. Sugahara KN, Hirata T, Hayasaka H, Stern R, Murai T, Miyasaka M. Tumor cells enhance their own CD44 cleavage and motility by generating hyaluronan fragments. J Biol Chem. 2006;281 (9):5861-8.

41. Duterme C, Mertens-Strijthagen J, Tammi M, Flamion B. Two novel functions of hyaluronidase-2 (Hyal2) are formation of the glycocalyx and control of CD44-ERM interactions. J Biol Chem. 2009;284(48):33495-508.

42. Marzese DM, Liu M, Huynh JL, Hirose H, Donovan NC, Huynh KT, Kiyohara E, Chong K, Cheng D, Tanaka R, Wang J, Morton DL, Barkhoudarian G, Kelly DF, Hoon DS. Brain metastasis is predetermined in early stages of cutaneous melanoma by CD44v6 expression through epigenetic regulation of the spliceosome. Pigment Cell Melanoma Res. 2015;28(1):82-93.

43. Zhang P, Fu C, Bai H, Song E, Song Y. CD44 variant, but not standard CD44 isoforms, mediate disassembly of endothelial VE-cadherin junction on metastatic melanoma cells. FEBS Lett. 2014;588(24):4573-82.

44. Petrey AC, de la Motte CA. Hyaluronan, a crucial regulator of inflammation. Front Immunol. 2014;5:101. 
45. Taylor KR, Yamasaki K, Radek KA, Di Nardo A, Goodarzi H, Golenbock D, Beutler B, Gallo RL. Recognition of hyaluronan released in sterile injury involves a unique receptor complex dependent on Toll-like receptor 4, CD44, and MD-2. J Biol Chem. 2007:282(25):18265-75.

46. de La Motte CA, Hascall VC, Calabro A, Yen-Lieberman B, Strong SA. Mononuclear leukocytes preferentially bind via CD44 to hyaluronan on human intestinal mucosal smooth muscle cells after virus infection or treatment with poly(l.C). J Biol Chem. 1999;274(43):30747-55.

47. Kessler S, Rho H, West G, Fiocchi C, Drazba J, de la Motte C. Hyaluronan $(\mathrm{HA})$ deposition precedes and promotes leukocyte recruitment in intestinal inflammation. Clin Transl Sci. 2008;1(1):57-61.

48. Gonda A, Gal I, Szanto S, Sarraj B, Glant TT, Hunyadi J, Mikecz K. CD44, but not l-selectin, is critically involved in leucocyte migration into the skin in a murine model of allergic dermatitis. Exp Dermatol. 2005;14(9):700-8.

49. McDonald B, McAvoy EF, Lam F, Gill V, de la Motte C, Savani RC, et al. Interaction of CD44 and hyaluronan is the dominant mechanism for neutrophil sequestration in inflamed liver sinusoids. J Exp Med. 2008;205(4):915-27.

50. McDonald B, Kubes P. Interactions between CD44 and Hyaluronan in Leukocyte Trafficking. Front Immunol. 2015;6:68.

51. Taylor RC, Patel A, Panageas KS, Busam KJ, Brady MS. Tumor-infiltrating lymphocytes predict sentinel lymph node positivity in patients with cutaneous melanoma. J Clin Oncol. 2007;25(7):869-75.

52. Thomas NE, Busam KJ, From L, Kricker A, Armstrong BK, Anton-Culver H, Gruber SB, Gallagher RP, Zanetti R, Rosso S, Dwyer T, Venn A, Kanetsky PA, Groben PA, Hao H, Orlow I, Reiner AS, Luo L, Paine S, Ollila DW, Wilcox H, Begg CB, Berwick M. Tumor-infiltrating lymphocyte grade in primary melanomas is independently associated with melanoma-specific survival in the population-based genes, environment and melanoma study. J Clin Oncol. 2013;31(33):4252-9.

53. Eggermont A, Robert C, Soria JC, Zitvogel L. Harnessing the immune system to provide long-term survival in patients with melanoma and other solid tumors. Oncoimmunology. 2014;3(1):e27560

\section{Submit your next manuscript to BioMed Central and we will help you at every step:}

- We accept pre-submission inquiries

- Our selector tool helps you to find the most relevant journal

- We provide round the clock customer support

- Convenient online submission

- Thorough peer review

- Inclusion in PubMed and all major indexing services

- Maximum visibility for your research

Submit your manuscript at www.biomedcentral.com/submit 